\title{
SACY: open clusters connected to young nearby associations
}

\author{
C. A. O. Torres, ${ }^{1}$ G. R. Quast, ${ }^{1}$ C. H. F. Melo,${ }^{2}$ M. Sterzik, ${ }^{2}$ \\ R. de la Reza ${ }^{3}$ and Licio da Silva ${ }^{3}$ \\ ${ }^{1}$ Laboratório Nacional de Astrofísica/MCT, Brazil \\ ${ }^{2}$ ESO, Santiago, Chile \\ ${ }^{3}$ Observatório Nacional/MCT, Brazil
}

\begin{abstract}
In recent years. we have spectroscopically observed thousands of counterparts of selected ROSAT bright sources in the SACY project (Search for Associations Containing Young stars). We demonstrated that hundreds of the young stars found this way belong to nearby young, loose associations, some detected only in SACY, with ages in the range from 5 to 100 Myr. For their ages, these associations show no trace of their primitive nurseries, which poses some difficulties as to their origins. Nevertheless, there are some associations clearly associated with young open clusters, such as the $\varepsilon$ Cha association (6 Myr) with the $\eta$ Cha cluster, and the Argus association with IC 2391 (40 Myr). There seem to exist also more subtle connections, such as the AB Dor association (75 Myr) with the Pleiades. Here, we present evidence of the connections of young associations to open clusters, to understand their origin and their possible insertion in the cluster infant-mortality and Gould Belt scenarios.
\end{abstract}

Keywords. open clusters and associations: individual ( $\eta$ Cha, $\varepsilon$ Cha, IC 2391, Argus association), solar neighborhood, stars: pre-main-sequence, stars: kinematics, surveys

The full poster (in pdf format) is available at http://www.astro.iag.usp.br/〜iaus266/Posters/pTorres.pdf. 